\title{
A Survey Report on Non-Dominationed Genetic Algorithm in Wireless Sensor Networks
}

\author{
Harjot Kaur \\ Research Scholar, \\ Shri Venkateshwara \\ University,Gajraula, India
}

\author{
Gaurav Tejpal, PhD \\ Professor, \\ Shri Venkateshwara University \\ Gajraula, India
}

\author{
Sonal Sharma,PhD \\ Assistant Professor, \\ Uttaranchal University \\ Dehradun, India
}

\begin{abstract}
In wanting to solve multiobjective optimization issues, many standard practices scalarize the aim vector right into a single objective. In these cases, the obtained alternative is extremely sensitive to the fat vector found in the scalarization method and requirements that the consumer have information about the main problem. Moreover, in solving multiobjective issues, manufacturers may be thinking about a set of Pareto-optimal factors, instead of a single point. Because genetic formulas (GAs) work with a populace of factors, it appears natural to utilize GAs in multiobjective optimization issues to fully capture several solutions simultaneously. While a vector evaluated GA (VEGA) has been executed by Schaffer and has been attempted to resolve several multiobjective issues, the algorithm seemingly have tendency toward some regions. In this report, we investigate Goldberg's concept of nondominated organizing in GAs and also a niche and speciation technique to get multiple Pareto-optimal factors simultaneously. The proof-of-principle effects obtained on three issues utilized by Schaffer and the others claim that the proposed technique can be extended to higher dimensional and more difficult multiobjective problems. A number of ideas for expansion and program of the algorithm will also be discussed.
\end{abstract}

\section{Keywords}

Coverage control Energy Multi-objective Genetic Algorithm in Wireless sensor network.

\section{INTRODUCTION}

Because of the restricted power and computational assets offered to indicator nodes, how many nodes stationed to cover the complete monitored region completely is usually higher than if your deterministic procedure were used. Activating only the mandatory quantity of indicator nodes at any particular moment is an effective way to save lots of the general power of the system. A book protection control scheme predicated on multi-objective genetic algorithm is proposed in this paper. The minimum quantity of detectors is picked in a densely stationed setting while preserving full coverage. Instead of the binary recognition indicator design in the last work, an even more precise recognition design is applied in conjunction with the protection control scheme. Simulation results show which our algorithm can achieve healthy efficiency on several types of recognition indicator models while maintaining large protection rate. With the same quantity of stationed detectors, our scheme compares positively with the existing schemes. With the rising demand for real time services in Wireless Sensor Systems (WSNs), quality of service (QoS) centered routing has surfaced as a fascinating research topic. But giving some QoS guarantee in indicator systems increases substantial challenges. The system wants to deal with battery limitations, while giving QoS (endto-end wait and reliability) guarantees. Developing such QoS routing practices that improve numerous objectives is computationally intractable. Higher energy exchange nodes can be used as chaos brains in a two-tiered WSN and these exchange nodes may sort a system among themselves to course data towards the sink. In this design, the QoS guarantee is set mostly by these exchange nodes. In this paper a solution predicated on NSGA-II is proposed for power effective QoS routing in chaos centered WSNs. Simulation results demonstrate that the proposed process outperforms system efficiency by optimizing numerous QoS variables and power consumption.

\section{CHARACTERSTICS OF GENETIC ALGORITHM}

The various features of Genetic Algorithm are Summarized as below (Goldberg 1999):

- Genetic Algorithm is on the basis of the notion of normal choice and normal genetics.

- They're straightforward and implement.

- They're thoroughly utilized in optimization problems.

- These work with citizenry of details as opposed to a person point.

- These use probabilistic change principle as opposed to deterministic rules.

- Genetic Methods may efficiently cope with big amount of variables.

- These methods don't involve any derivative information.

- They're far better for complex problems than easy issues. 


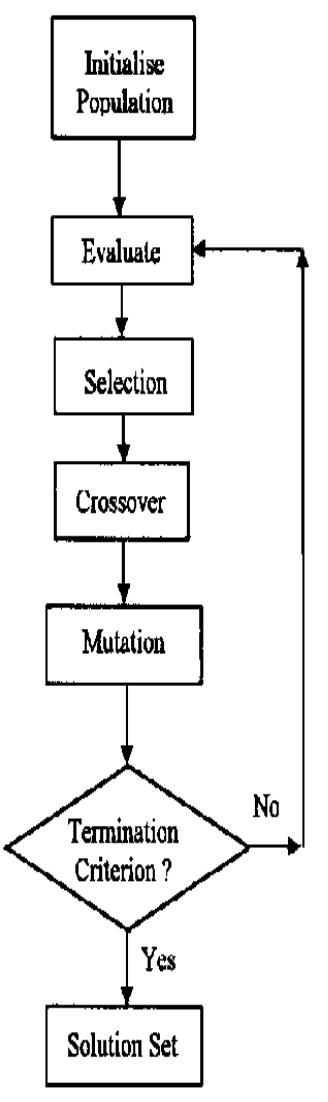

Fig 1: Shows application of genetic algorithm

\section{APPLICATIONS OF GENETIC ALGORITHM \\ 3.1 Radiology}

Imaging practices in radiology create a wide range of information that requires to be reviewed and saw by radiologists in a somewhat small time. Computer-aided recognition and examination are quickly rising interdisciplinary systems that purpose to help radiologists in quicker and more exact picture examination by recognition, segmentation, and classification of typical and pathological designs entirely on numerous imaging modalities. These generally include $\mathrm{X}$-rays, magnetic resonance imaging (MRI), compute tomography (CT) check, and ultrasound. ${ }^{13}$

\subsection{Optimized Telecommunications Routing}

Do you will find yourself annoyed by gradual LAN efficiency, contradictory access to the internet, a FAX device that just directs faxes often, your area line's amount of 'cat'calls on a monthly basis? Effectively, GAs are increasingly being created which will permit active and anticipatory redirecting of tracks for communities. These might take detect of one's system's instability and assume your re-routing needs. Applying multiple GA circuit-search at the same time, shortly your social communications issues might actually be all in your mind as opposed to in your telecommunications system. Different GAs are increasingly being created to improve place and redirecting of mobile systems for most readily useful protection and simple changing, which means that your cellular phone and rim will undoubtedly be glad for GAs also.

\subsection{Trip, Traffic and Shipment Routing}

New programs of a GA referred to as the "Touring Jeweler Problem" or TSP can be utilized to approach the absolute most successful avenues and arrangement for journey planners, traffic modems and also delivery companies. The quickest avenues for traveling. The moment to prevent traffic tie-ups and run hours. Many successful usage of transportation for delivery, to even including collection masses and deliveries over the way. This system could be modeling all of this in the back ground whilst the individual brokers do other items, increasing production as properly! Odds are raising gradually that whenever you obtain that journey approach package from the journey company, a GA added more to it compared to representative did.

\subsection{Optimization}

Genetic Methods are many typically found in optimization issues when we've to maximise or reduce certain target purpose price below certain group of constraints. The strategy to resolve Optimization issues has been outlined through the entire tutorial.

\subsubsection{Question Optimization}

Question is definitely an fundamental part of a spread repository system. Generally speaking, it's applied to effectively attain various procedures of the repository objects. Question optimization is a procedure that creates different function website allocation programs to accomplish the query. Function website allocation strategy presents the device of question execution. The aim of function website allocation issue is to choose an optimum question performance strategy which optimizes the Full Prices of the spread choice help program query. Amount of experts have optimizes the question applying genetic algorithm and their variation. They discovered that GA increases results than different oldfashioned methods since it allows the perfect answer really rapidly $[1 / 2 / 3 / 4 / 5]$.

\subsection{Similar Handling}

Similar handling is definitely an effective strategy to generally meet the computational demands of the big quantity of problems. Plenty of experts are picking out improvements and providing new some ideas for the development. In similar handling, among the significant issues could be the arrangement of responsibilities to different devices or nodes. Variety of study have attempted to examine use of similar handling to reduce the make span of the task. Writers elucidated that the processors and connection hyperlinks are essential sources in similar processing techniques and their effective administration through appropriate arrangement is required for obtaining large performance.

\subsection{Mutation}

A gene mutation is a lasting modification in the DNA routine which makes up a gene, in a way that the routine varies from what's present in many people. Mutations selection in dimensions; they could influence everywhere from an individual DNA making stop (base pair) to a sizable part of a chromosome that features numerous genes.

\subsection{Crossover}

In genetic calculations, crossover is a genetic user applied to alter the coding of a chromosome or chromosomes from era to the next. It's comparable to natural crossover, upon which 
genetic calculations are based. Crossover is an activity of getting multiple parent alternatives and providing a kid alternative from their store.

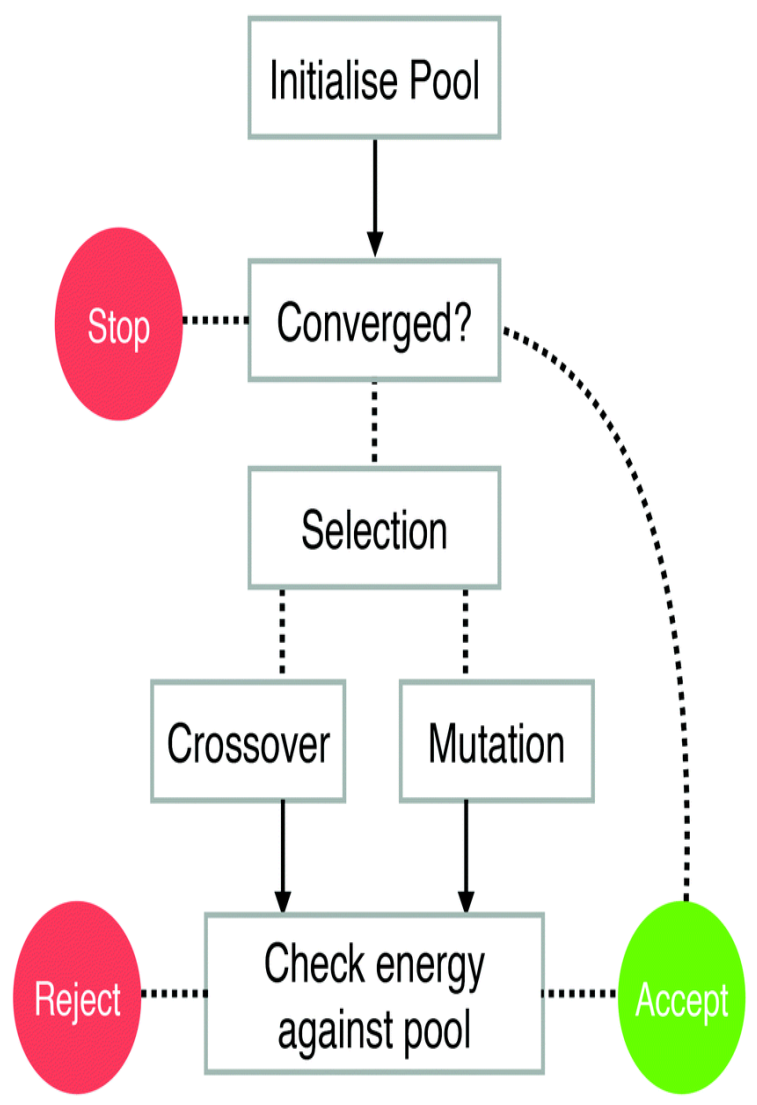

Fig 1: Represent the Diagram of Crossover and Mutation

\section{CONCLUSION}

In this report, we investigate Goldberg's concept of no dominated organizing in GAs and also a niche and speciation technique to get multiple Pareto-optimal factors simultaneously. The proof-of-principle effects obtained on three issues utilized by Schaffer and the others claim that the proposed technique can be extended to higher dimensional and more difficult multiobjective problems. A number of ideas for expansion and program of the algorithm will also be discussed.

\section{REFERENCES}

[1] Deb, Kalyanmoy, et al. "A fast elitist non-dominated sorting genetic algorithm for multi-objective optimization: NSGA-II." International Conference on Parallel Problem Solving From Nature. Springer, Berlin, Heidelberg, 2000.

[2] Srinivas, Nidamarthi, and Kalyanmoy Deb. "Muiltiobjective optimization using nondominated sorting in genetic algorithms." Evolutionary computation 2.3 (1994): 221-248.

[3] Deb, Kalyanmoy, and Tushar Goel. "Controlled elitist non-dominated sorting genetic algorithms for better convergence." Evolutionary multi-criterion optimization. Springer Berlin/Heidelberg, 2001.
[4] Deb, Kalyanmoy, et al. "A fast and elitist multiobjective genetic algorithm: NSGA-II." IEEE transactions on evolutionary computation 6.2 (2002): 182-197.

[5] Milosevic, Borka, and Miroslav Begovic. "Nondominated sorting genetic algorithm for optimal phasor measurement placement." IEEE Transactions on Power Systems 18.1 (2003): 69-75.

[6] DEH, Kalyanmoy, et al. "A fast elitist non-dominated sorting genetic algorithm for multi-objective optimization: NSGA-II." Lecture notes in computer science(2000): 849-858.

[7] Li, Xiaodong. "A non-dominated sorting particle swarm optimizer for multiobjective optimization." Genetic and Evolutionary Computation-GECCO 2003. Springer Berlin/Heidelberg, 2003.

[8] Kanagarajan, D., et al. "Optimization of electrical discharge machining characteristics of $\mathrm{WC} / \mathrm{Co}$ composites using non-dominated sorting genetic algorithm (NSGA-II)." The International Journal of Advanced Manufacturing Technology 36.11-12 (2008): 1124-1132.

[9] Basu, M. "Dynamic economic emission dispatch using nondominated sorting genetic algorithm-II." International Journal of Electrical Power \& Energy Systems 30.2 (2008): 140-149.

[10] Sun, Dazhi, Rahim F. Benekohal, and S. Travis Waller. "Multiobjective traffic signal timing optimization using non-dominated sorting genetic algorithm." Intelligent vehicles symposium, 2003. proceedings. ieee. IEEE, 2003.

[11] Guria, Chandan, Prashant K. Bhattacharya, and Santosh K. Gupta. "Multi-objective optimization of reverse osmosis desalination units using different adaptations of the non-dominated sorting genetic algorithm (NSGA)." Computers \& chemical engineering 29.9 (2005): 1977-1995.

[12] Cao, Kai, et al. "Spatial multi-objective land use optimization: extensions to the non-dominated sorting genetic algorithm-II." International Journal of Geographical Information Science 25.12 (2011): 19491969.

[13] Panda, Sidhartha. "Multi-objective PID controller tuning for a FACTS-based damping stabilizer using Nondominated Sorting Genetic Algorithm-II." International Journal of Electrical Power \& Energy Systems 33.7 (2011): 1296-1308

[14] Wang, Long, Tong-guang Wang, and Yuan Luo. "Improved non-dominated sorting genetic algorithm (NSGA)-II in multi-objective optimization studies of wind turbine blades." Applied Mathematics and Mechanics 32.6 (2011): 739-748.

[15] Ghoddousi, Parviz, et al. "Multi-mode resourceconstrained discrete time-cost-resource optimization in project scheduling using non-dominated sorting genetic algorithm." Automation in construction 30 (2013): 216227.

[16] Mandal, Debabrata, Surjya K. Pal, and Partha Saha. "Modeling of electrical discharge machining process using back propagation neural network and multiobjective optimization using non-dominating sorting 
genetic algorithm-II." Journal of Materials Processing Technology 186.1 (2007): 154-162.

[17] Zhihuan, Li, Li Yinhong, and Duan Xianzhong. "Nondominated sorting genetic algorithm-II for robust multiobjective optimal reactive power dispatch." IET generation, transmission \& distribution 4.9 (2010): 10001008 .

[18] Nandasana, Anjana D., Ajay Kumar Ray, and Santosh K. Gupta. "Applications of the Non-Dominated Sorting Genetic Algorithm(NSGA) in Chemical Reaction Engineering." International Journal of Chemical and Reactor Engineering 1 (2003): 1018.
[19] Inamdar, S. V., Santosh K. Gupta, and D. N. Saraf. "Multi-objective optimization of an industrial crude distillation unit using the elitist non-dominated sorting genetic algorithm." Chemical Engineering Research and Design 82.5 (2004): 611-623.

[20] Yang, S. H., and U. Natarajan. "Multi-objective optimization of cutting parameters in turning process using differential evolution and non-dominated sorting genetic algorithm-II approaches." The International Journal of Advanced Manufacturing Technology 49.5 (2010):773-78. 